\title{
Automedicação na adolescência: um desafio para a educação em saúde
}

\author{
Self-medication in adolescence: a challenge to health education
}

Ilane $M$ agalhães Silva ${ }^{1}$

Ana M aria Fontenelle Catrib ${ }^{1}$

Vânia Cordeiro de $M$ atos ${ }^{1}$

Ana Paula Soares Gondim ${ }^{1}$

${ }^{1}$ Universidade de Fortaleza. Av. Washington Soares 1.321, Edson Queiroz. 60811-341 Fortaleza CE. ilane_mag@yahoo.com.br
Abstract The study aims to examine the knowledge of students of public and private schools in the city of Fortaleza, Ceará State, Brazil on the use of medicine and their health implications. It is a descriptive study of 722 adolescents enrolled in ten schools in the city of Fortaleza. He got up aspects of consumption, indication and guidance medicineand influence of themedia using a questionnaire. Seventy two percent of the participants reported use of medicine the last 60 days, and painkillers were the most cited $(65.4 \%)$. The indications were responsible for the family ( $51.2 \%$ ) and physicians (33.1\%), and the self-medication by $20.8 \%$ of the students. Regarding the use of medicine $70.9 \%$ of thestudents were not instructed on the proper use of the medicine. Of the total, $34.1 \%$ had already been influenced by media in the purchase of medicine. It's possible to conclude that teenagers' knowledge about medi cineand its implications on health is very weak and devoid of any basic notion of the rational use of medicine. In addition, public school students are more oriented on the proper use of medicines by professionals in the public health system, which demonstrates the efficiency of the promoter of health of the population enrolled in public schools in Fortaleza.

Key words Self-medication, Adolescents, Health education
Resumo 0 estudo visa analisar o conhecimento dos estudantes de escolas públicas e privadas do município de Fortaleza (CE) sobre o uso de medicamentos e suas implicações para a saúde. Tratase de um estudo descritivo, com 722 adolescentes matriculados em dez escolas do município deFortaleza. Levantou-se, por meio de um questionário, aspectos relativos ao consumo, indicação e orientação de medi camentosea influência da mídia. Relataram uso demedicamentos, nosúltimos sessenta dias, 72,0\% dos participantes, sendo os analgési cos os mais citados (65,4\% ). O s responsáveis pelas indicações foram familiares $(51,2 \%)$ e médicos $(33,1 \%)$, sendo a automedicação relatada por $20,8 \%$ dos estudantes. Não receberam orientações sobre o uso correto de medicamentos $70,9 \%$ dos alunos. Do total, 34,1\% revelaram já terem sido influenciados por meios de comunicação na compra de medicamentos. É possível concluir que o conhecimento dos adolescentes sobre medicamentos e suas implicações na saúde é bastante incipiente e desprovido de qualquer noção básica sobre o uso racional deles. Além disso, os estudantes da rede pública são mais orientados sobre o uso correto de medicamentos, por parte dos profissionais do sistema público de saúde, 0 que demonstra a eficiência do sistema como promotor desaúde da população matriculada em escolas públicas de Fortaleza.

Palavras-chave Automedicação, Adolescentes, Educação em saúde 


\section{Introdução}

A saúde deixou deser uma premissa existencial do ser humano, ao tornar-se campo da atividade econômica, decorrente de mudanças estruturais implantadas, objetivando a ampliação de mercado ${ }^{1}$. Segundo a Sociedade Brasileira de Vigilância de M edicamentos (Sobravime) ${ }^{2}$, o medicamento como parte do complexo médico industrial influi na percepção da saúdee da doença, tanto nos profissionais da saúde quanto na sociedade. 0 medicamento não se apresenta sozinho - como substância química - mas está acompanhado por um cortejo de publicidade, informação, brindes, estudos, etc., configurando uma forma de pensar.

$A$ automedicação é definida como o uso de medicamentos sem prescrição médica, sendo 0 próprio pacientequem decidequal éo fármaco a ser utilizado, com o objetivo de tratar ou aliviar sintomas ou mesmo de promover a saúde, independentemente da prescrição profissional ${ }^{3}$. Os medicamentos possuem forte valor simbólico para a população, transcendendo a sua atividade terapêutica, o que contribui para maior consumo e uso irracional destes 4 .

Consoante Lage et al. ${ }^{5}$, os medicamentos são reconhecidos como instrumentos indispen sáveis às ações de saúde, ocupando papel central na terapêutica da atualidade. Conforme os mesmos autores, os fatores relacionados ao modo e utilização do medicamento refletem-se no efeito terapêutico. Bem assim, a orientação recebida no consultório médico é confrontada com outras fornecidas por diferentes profissionais da área da saúde, com aquelas obtidas por parentes, vizinhos e, também, nos meios de comunicação social. Esses confrontos podem levar ao uso inadequado desses produtos.

Os elevados índices de prescrição de antibióticos, a carência de orientação dos usuários por parte dos profissionais prescritores e dispensadores, as publicidades diretas ao consumidor final e o uso incorreto por parte do paciente constituem barreiras para a promoção do uso racional de medicamentos. No Brasil, há outros fatores que agravam este quadro; cerca de oitenta milhões de pessoas praticam a automedicação, há uma má qualidade de oferta de medicamentos, não se cumpre a obrigatoriedade da receita médica e há uma carência de informações e instrução da população em geral, o que justifica a preocupação em implementar as estratégias do uso racional de medicamentos ${ }^{6,7}$.

Visando minimizar os fatores citados, a Organização Mundial Saúde (OMS) ${ }^{8}$ estabeleceu como meta para a avaliação do uso de medicamentos e controle do seu consumo, com o objetivo de melhorar a racionalidade do uso nas próximas décadas.

Os estudos sobre automedicação em crianças e adolescentes brasileiros foram desenvolvidos em serviços de saúde ou constituíram-se em estudos de base populacional, sendo esses: (1) epidemiologia do consumo demedicamentosem crianças de centro urbano da região sul do Brasil $^{9}$; (2) automedicação em pediatria geral ${ }^{10}$; (3) utilização de medicamentos por crianças atendidas em creches ${ }^{11}$; (4) epidemiologia do consumo de medicamentos no primeiro trimestre de vida em centro urbano do sul do Brasil ${ }^{12 ;}$; (5) estudo da automedicação infantil em uma região administrativa no município do Rio de Janeiro ${ }^{13}$; (6) uso judicioso de medicamentos em crianças $^{14} \mathrm{e}$ (7) automedicação em crianças e adolescente ${ }^{15}$. 0 que diferencia esta pesquisa das demais é 0 fato de ter sido realizada na escola, espaço quese apresenta apropriado para desenvolver ações de saúdee veicular informações edu cativas junto aos adolescentes, na tentativa de expandir saberes e conhecimentos de forma efetiva e abrangente sobre o uso racional de medicamentos.

Outra consideração importante é o fato de que o adolescenteéportador de uma experiência de vida e se encontra formulando socialmente seu ponto de vista sobre determinados saberes, podendo este disseminar o conhecimento sobre o uso racional de medicamentos junto à família, amigos e sociedade.

Com base nestas considerações, este estudo questionou os adolescentes sobre a automedicação e a influência dos meios de comunicação, abordando, também, aspectos relativos ao consumo de medicamentos, os responsáveis pelas indicações epelas orientações dadas sobre os fármacos utilizados.

$\mathrm{Na}$ tentativa de contribuir para $\mathrm{o}$ aprimoramento da temática, este ensai o deteve- seem analisar o conhecimento dos estudantes de escolas públicas e privadas do município de Fortaleza (CE) sobre o uso de medicamentos e suas implicações para a saúde.

\section{Metodologia}

Trata-se de um estudo transversal, de abordagem quantitativa. A pesquisafoi realizada em dez escolas do município de Fortaleza, sendo cinco mantidas por iniciativa pública ecinco privadas. No projeto inicial, estava prevista a inclusão de 
doze escolas (seis públicas e seis privadas), re presentando as seis Secretarias Executivas Regionais (SER) do município deFortaleza; no entanto, por motivos de recusa de duas escolas, a pesquisa foi realizada em dez unidades escolares.

Os estabelecimentos de ensino estavam cadastrados em diferentes SER e foram tomadas al eatoriamente cinco destas secretarias, contanto que cada secretaria regional fosse contemplada por uma escola pública e uma particular que oferecessem ensino fundamental e médio.

A amostra constituiu-se de 722 adolescentes escolares, baseada na prevalência de automedicação em criança $(41,4 \%)^{13}$. Foram escolhidos aleatoriamente alunos de treze a dezoito anos, matriculados na educação básica nas diferentes SER que aceitaram participar da investigação e encontravam-se presentes no momento da aplicação do instrumento de coleta de dados. No ano de 2006, o município de Fortaleza contava com 7.675 alunos matriculados nas dez escolas de educação básica.

A coleta de dados foi realizada de setembro de 2006 a outubro de 2007, usando-se um questionário contendo sete questões fechadas e cinco abertas, que abordavam aspectos relacionados às características soci odemográficas (idade, sexo, dependência administrativa, série, turno), conceito e finalidade de medicamento, justificativa de uso de medicamento sem receita médica, medicamentos utilizados nos últimos sessenta dias, responsáveis pela indicação e orientação sobre 0 uso correto destes, influência dos meios de comunicação, uso diário de medicamento em casa, transporte de medicamento para uso próprio, uso de medicamento a partir da leitura de bulas, uso de medicamento sem consulta médica, classes terapêuticas mais utilizadas euso e indicação de antimicrobianos.

Para a montagem do banco de dados e análise dos resultados, utilizou-se o programa estatístico SPSS, versão 11.0. Realizou-se uma análise estatística descritiva simples. Para as variáveis categóricas, foi realizada uma análise de frequência absoluta e relativa, cujos resultados foram apresentados em forma de tabelas, considerando-se dois grupos: escola pública e escola privada.

Em atendimento a Resolução n 196/96, do Conselho Nacional de Saúde ${ }^{16}$, que preconiza e regulamenta as pesquisas envolvendo seres humanos, esta pesquisa foi aprovada pelo Comitê de Ética e Pesquisa da UNIFOR. A autorização para aplicar o questionário foi obtido por meio do termo de consentimento livre e esclarecido, após apresentação e esclarecimento de todo 0 projeto aos adolescentes escolares ou responsáveis por eles.

\section{Resultados}

Participaram do estudo 722 adolescentes, sendo $450(62,3 \%)$ estudantes de escolas mantidas por iniciativa pública e $272(37,7 \%)$ de escolas privadas. 0 sexo feminino foi predominante, com 393 $(54,4 \%)$ estudantes. Quanto à idade dos alunos, a faixa de variação foi de treze a dezoito anos ea maioria de $641(88,7 \%)$ tinhaidade entrequinzee dezoito anos. Quanto à sérieem curso, $63(8,7 \%)$, $287(39,8 \%), 191(26,5 \%)$ e $181(25,1 \%)$, correspondem, respectivamente, ao 9o ano do ensino fundamental, 1ㅇ, 2ㅇ e 3ㅇ anos do ensino médio. Estudavam no turno da manhã 596 (82,5\%) participantes e $126(17,5 \%)$, no turno da tarde.

O bserva-se na Tabela 1 que 320 (46,9\%) alunos definiram o medicamento como "substância química com objetivo decura" e215 (31,5\%) conceituaram como "método de aliviar as doenças". Houve $56(8,2 \%)$ alunos que denotaram o significado como "meio de combater sintomas de uma doença" e dezenove $(2,8 \%)$ como "útil para prevenir doenças". As proporções dos conceitos dados por alunos de escolas públicas e privadas apresentaram semelhança de acordo com as respostas dos estudantes, nas quais se observa a ideia de cura associada ao uso do medicamento, cuja ingestão é referida como responsável pela melhoria do corpo acometido pelas doenças.

$\mathrm{Na}$ Tabela 1, também é possível observar as opiniões dos alunos referentes ao uso de medicamentos sem receita médica. D entre os 669 participantes que responderam à pergunta, 114 $(17,0 \%)$ acreditavam que isso ocorre porque as pessoas já possuem experiência com o medicamento, $97(14,5 \%)$ defendiam a ideia de que a falta de informação e/ou orientação leva à realização de tal prática, 91 (13,6\%) citaram a influência da televisão e amigos, 82 (12,3\%) relataram a preguiça deir ao médico ou pela praticidade, sendo essa resposta mais frequente em alunos das escolas privadas. A dificuldade no atendimento do SUS foi outra razão dada por 77 $(11,5 \%)$ estudantes, principalmente aqueles de escola pública.

Quando questionados se podiam tomar medicamentos por conta própria, 393 responderam que "não". As principais justificativas foram poder apresentar efeitos adversos ou contrários do medicamento, com 96 citações, e causar danos à saúde, com sessenta, sendo a primeira afirmação a 
mais citada entre alunos de escolas públicas e a segunda a mais mencionada entre alunos de escolas privadas. Dos que afirmaram que podem tomar medicamentos por conta própria, 153 justificaram, relatando ser algo simples o que eles sentiam, 74 não justificaram as respostas, 54 citaram outras justificativas, onze o fazem porque possuem dificuldades de marcar a consulta (Tabela 1).
Em suas respostas, os estudantes da pesquisa revelaram que o custo elevado dos medicamentos prescritos pelos médicos e a dificuldade de acesso aos postos de saúde são fatores determinantes para a automedicação, como, também, demonstraram a banalização com que se referem aos medicamentos e a facilidade com que os adquirem sem a orientação de um profissional.

Tabela 1. Conhecimento dos estudantes sobre medicamentos e automedicação.

\begin{tabular}{|c|c|c|c|c|c|c|}
\hline \multirow{3}{*}{ Variáveis } & & & \multicolumn{4}{|c|}{ Dependência administrativa } \\
\hline & \multicolumn{2}{|c|}{ Total } & \multicolumn{2}{|c|}{ Públicas } & \multicolumn{2}{|c|}{ Privadas } \\
\hline & $\mathrm{n}$ & $\%$ & $\mathrm{n}$ & $\%$ & $\mathrm{n}$ & $\%$ \\
\hline O que é e para que serve o medicamento?* & 682 & 100 & 423 & 100 & 259 & 100 \\
\hline Substância química com objetivo de cura & 320 & 46,9 & 220 & 52,0 & 100 & 38,6 \\
\hline M étodo de aliviar as doenças & 215 & 31,5 & 90 & 21,3 & 125 & 48,3 \\
\hline Combater os sintomas de uma doença & 56 & 8,2 & 54 & 12,8 & 02 & 0,8 \\
\hline útil para prevenir doenças & 19 & 2,8 & 14 & 3,3 & 05 & 1,9 \\
\hline Outros & 72 & 10,6 & 45 & 10,6 & 27 & 10,4 \\
\hline $\begin{array}{l}\text { Por que as pessoas tomam medicamentos sem } \\
\text { receita médica?* }\end{array}$ & 669 & 100 & 414 & 100 & 255 & 100 \\
\hline Experiência com o medicamento & 114 & 17,0 & 72 & 17,4 & 42 & 16,5 \\
\hline Falta de informação e/ou orientação & 97 & 14,5 & 58 & 14,0 & 39 & 15,3 \\
\hline Influência da TV e amigos & 91 & 13,6 & 59 & 14,2 & 32 & 12,5 \\
\hline Preguiça de ir ao médico/praticidade & 82 & 12,3 & 36 & 8,7 & 46 & 18,0 \\
\hline Dificuldade no atendimento do SUS & 77 & 11,5 & 72 & 17,4 & 05 & 2,0 \\
\hline Falta de acesso ao médico & 65 & 9,7 & 45 & 10,9 & 20 & 7,8 \\
\hline Necessidade de uma melhora imediata & 57 & 8,5 & 36 & 8,7 & 21 & 8,2 \\
\hline N ão acham necessário & 16 & 2,4 & 00 & 0,0 & 16 & 6,3 \\
\hline Não sabiam & 09 & 1,3 & 00 & 0,0 & 09 & 3,5 \\
\hline Costume & 07 & 1,1 & 00 & 0,0 & 07 & 2,7 \\
\hline Outros & 54 & 8,1 & 36 & 8,7 & 18 & 7,1 \\
\hline Você pode tomar medicamentos por conta própria?* & 693 & 100 & 432 & 100 & 261 & 100 \\
\hline Não & 393 & 56,7 & 270 & 62,5 & 123 & 47,1 \\
\hline Sim & 300 & 43,3 & 162 & 37,5 & 138 & 52,9 \\
\hline \multicolumn{7}{|l|}{ J ustificativas para a resposta NÃO } \\
\hline A presentar efeito adverso ou contrário & 96 & 24,4 & 84 & 31,1 & 12 & 9,8 \\
\hline Causar danos à saúde & 60 & 15,3 & 35 & 13,0 & 25 & 20,3 \\
\hline Não justificaram & 56 & 14,3 & 24 & 8,9 & 32 & 26,0 \\
\hline Haver a possibilidade de tomá-lo errado & 44 & 11,2 & 32 & 11,8 & 12 & 9,8 \\
\hline Piorar os sintomas da doença & 43 & 10,9 & 41 & 15,2 & 02 & 1,6 \\
\hline Por não saber o efeito & 22 & 5,6 & 08 & 3,0 & 14 & 11,4 \\
\hline Ter a necessidade de orientação adequada & 22 & 5,6 & 14 & 5,2 & 08 & 6,5 \\
\hline Outros & 50 & 12,7 & 32 & 11,8 & 18 & 14,6 \\
\hline \multicolumn{7}{|l|}{ J ustificativas para a resposta SI M } \\
\hline Por ser algo simples & 153 & 51,0 & 81 & 50,0 & 72 & 52,1 \\
\hline Não justificaram & 74 & 24,7 & 30 & 18,5 & 44 & 31,9 \\
\hline Dificuldade de marcar consulta & 11 & 3,7 & 09 & 5,6 & 02 & 1,4 \\
\hline Por que não tenho alergia & 08 & 2,6 & 07 & 4,3 & 01 & 0,7 \\
\hline Outros & 54 & 18,0 & 35 & 21,6 & 19 & 13,8 \\
\hline
\end{tabular}

Fonte: Dados originais.

*D o total de participantes $(n=722)$, foram excluídos aqueles que não responderam à pergunta. 
Pode-se observar que, dos participantes do estudo, 520 (72,0\%) tinham feito uso de um ou mais medicamentos nos últimos sessenta dias, com predominância do sexo feminino $(60,0 \%)$. Houve maior consumo por alunos da rede privada de ensino. $\mathrm{Na}$ Tabela 2, dentre as classes de medicamentos mais citadas, estavam os analgé sicos, com $340(65,4 \%)$ relatos, os antigripais, com 64 (12,3\%), os antitérmicos, com 61 (11,7\%) e os antimicrobianos, com 51 (9,8\%). Acrescenta-se que os alunos referiram o uso de uma variedade de outros medicamentos com diversas indicações terapêuticas. As classes medicamentosas foram contabilizadas somente uma vez, nos casos em que foram utilizados dois ou mais medicamentos da mesma classe por alunos de escolas públicas e privadas.

Dos que fizeram uso de medicamentos, 266 $(51,2 \%)$ tiveram indicações por parte defamilia- res, 172 (33,1\%) demédicose 108 (20,8\%) foram por conta própria. A indicação por médicos (38,9\%) e a automedicação $(28,1 \%)$ foram maiores para alunos das escolas privadas em termos percentuais (Tabela 2).

Do total dos estudantes, $512(70,9 \%)$ não receberam ou não se lembram de haver recebido orientações sobre o uso correto de medicamentos. Dos queforam orientados, $91(43,3 \%)$ receberam a orientação de médicos, $82(39,0 \%)$ de familiares, $44(21,0 \%)$ na escola e $33(15,7 \%)$ no posto de saúde. Os alunos de escolas do sistema público foram menos orientados pelos familiares e escolas, porém mais orientados por médicos do que os al unos de escolas da rede privada.

A Tabela 3 relata a influência que os meios de comunicação exerceram sobreos adolescentes no momento da escolha de um medicamento. Dos estudantes, $246(34,1 \%)$ relataram quejá fizeram

Tabela 2. Distribuição das respostas dos adolescentes relativas ao uso de medicamentos, indicações e orientações.

\begin{tabular}{|c|c|c|c|c|c|c|}
\hline \multirow{3}{*}{ Variáveis } & \multirow{2}{*}{\multicolumn{2}{|c|}{ Total }} & \multicolumn{4}{|c|}{ Dependência administrativa } \\
\hline & & & \multicolumn{2}{|c|}{ Públicas } & \multicolumn{2}{|c|}{ Privadas } \\
\hline & $\mathrm{n}$ & $\%$ & $n$ & $\%$ & $\mathrm{n}$ & $\%$ \\
\hline \multicolumn{7}{|c|}{ Classes de medicamentos mais utilizadas* } \\
\hline a) Analgésicos & 340 & 65,4 & 192 & 64,2 & 148 & 67,0 \\
\hline b) Antigripais & 64 & 12,3 & 39 & 13,0 & 25 & 11,3 \\
\hline c) Antitérmicos & 61 & 11,7 & 42 & 14,0 & 19 & 8,6 \\
\hline d) Antimicrobianos & 51 & 9,8 & 32 & 10,7 & 19 & 8,6 \\
\hline e) Polivitamínicos & 23 & 4,4 & 12 & 4,0 & 11 & 5,0 \\
\hline f) Anti-inflamatórios & 20 & 3,8 & 14 & 4,7 & 06 & 2,7 \\
\hline g) Vermífugos & 12 & 2,3 & 09 & 3,0 & 03 & 1,4 \\
\hline h) Antialérgicos & 11 & 2,1 & 02 & 0,7 & 09 & 4,1 \\
\hline i) Anticoncepcionais & 10 & 1,9 & 07 & 2,3 & 03 & 1,4 \\
\hline j) Antianêmicos & 08 & 1,5 & 07 & 2,3 & 01 & 0,5 \\
\hline I) Não lembram o nome & 17 & 3,3 & 12 & 4,0 & 05 & 2,3 \\
\hline m) Outras classes & 93 & 17,9 & 45 & 15,1 & 48 & 21,7 \\
\hline n) Não responderam & 21 & 4,0 & 12 & 4,0 & 09 & 4,1 \\
\hline \multicolumn{7}{|l|}{ Quem indicou* } \\
\hline a) Familiares & 266 & 51,2 & 154 & 51,5 & 103 & 46,6 \\
\hline b) M édico & 172 & 33,1 & 86 & 28,8 & 86 & 38,9 \\
\hline c) Por conta própria & 108 & 20,8 & 46 & 15,4 & 62 & 28,1 \\
\hline d) Amigos & 33 & 6,3 & 21 & 7,0 & 12 & 5,4 \\
\hline e) Farmacêutico & 20 & 3,8 & 13 & 4,3 & 07 & 3,2 \\
\hline f) Meios de comunicação & 13 & 2,5 & 09 & 3,0 & 04 & 1,8 \\
\hline g) Balconista & 03 & 0,6 & 03 & 1,0 & 00 & 0,0 \\
\hline h) Outros & 05 & 1,0 & 04 & 1,3 & 01 & 0,5 \\
\hline i) N ão responderam & 30 & 5,8 & 19 & 6,4 & 11 & 5,0 \\
\hline
\end{tabular}

Fonte: Dados originais.

"Os participantes poderiam ter feito uso de um ou mais medicamentos eoptado por mais de uma resposta. Incluem os participantes queafirmaram "sim" na pergunta anterior. 
uso de medicamentos influenciados pela mídia, sendo a televisão (TV) a mais citada, com 229 $(93,1 \%)$ relatos, seguida dosjornaise revistas, com $05(2,0 \%)$, o rádio, com $03(1,2 \%)$, e outros, como, por exemplo, a Internet, com nove (3,7\%). Quase a totalidade de adolescentes se mostrou influenciada pelos meios de comunicação.

Destaca-se que o sexo feminino mostra-se mais influenciado pela mídia. Das $126(24,2 \%)$ adolescentes que utilizaram medicamentos para cólica, $76(60,3 \%)$ fizeram uso do medicamento composto "dipirona sódica + caféna anidra + citrato de orfenadrina" que, durante o período da coleta de dados, realizava o seu marketing através da mídia tel evisiva. Estes eram tomados, sem indicação, em 24 (31,6\%) casos. Os familiares indicaram o medicamento "dipirona sódica + cafeína anidra + citrato de orfenadrina" em 21 (27,6\%) casos (Tabela 3).

Quanto ao uso de antimicrobianos, 51 $(9,8 \%)$ utilizaram essa classe de medicamentos, sendo o médico e familiares os maiores responsáveispela indicação destes, com 22 casos (43,1\%) cada um, seguidos da automedicação, com sete eventos $(13,8 \%)$. As enfermidades mais relatadas pelos alunos foram dor na garganta ou virose $(45,1 \%)$ e inflamação $(25,5 \%)$. Os estudantes de escolas públicas tiveram maior número de indicações por parte dos familiares, ao contrário dos estudantes das escolas privadas, que tiveram um maior número de indicações por parte dos médicos (Tabela 4).

Em os locais mais citados para obtenção de medicamentos sem receita médica, 471 (66,2\%) citaram a farmácia, $277(38,9 \%)$ relataram conseguir em casa, 135 (19,0\%) adquiriam com amigos, $122(17,1 \%)$ obtinham com o médico ou posto de saúde, $121(17,0 \%)$ compravam em supermercados/mercearias, havendo diferença significativa, em termos percentuais, entre alunos de escolas públicas $(25,8 \%)$ e privadas $(2,6 \%)$. Do restante, quinze $(2,1 \%)$ obtinham os medicamentos nas escolas e trinta $(4,2 \%)$ os adquiriam em outros locais. Dez participantes não responderam a esta questão; portanto, o total re presenta 712 sujeitos, que puderam optar por mais de uma resposta.

D os estudantes, $314(44,2 \%)$ ressaltaram que sempre liam a bula, $308(43,3 \%)$ realizavam essa prática eventualmente e 89 (12,5\%) jamais liam. No queconcerneao entendimento da orientação da bula, $329(46,3 \%)$ responderam que entendiam bem, 44 (6,2\%) não entendiam, 292 (41,1\%) entendiam às vezes e $45(6,3 \%)$ realmente não liam a bula. A maioria, 549 (76,8\%) alunos, verificava a data de validade dos medicamentos.

Tabela 3. Influência dos meios de comunicação na automedicação.

\begin{tabular}{|c|c|c|c|c|c|c|}
\hline \multirow{3}{*}{ Variáveis } & & & \multicolumn{4}{|c|}{ Dependência administrativa } \\
\hline & \multicolumn{2}{|c|}{ Total } & \multicolumn{2}{|c|}{ Públicas } & \multicolumn{2}{|c|}{ Privadas } \\
\hline & $\mathrm{n}$ & $\%$ & $n$ & $\%$ & $\mathrm{n}$ & $\%$ \\
\hline \multicolumn{7}{|l|}{ Qual o meio de comunicação?* } \\
\hline a) TV & 229 & 93,1 & 158 & 93,5 & 71 & 92,2 \\
\hline b) Jornais e revistas & 05 & 2,0 & 04 & 0,9 & 01 & 1,3 \\
\hline c) Rádio & 03 & 1,2 & 02 & 1,2 & 01 & 1,3 \\
\hline d) Outros & 09 & 3,7 & 05 & 1,1 & 04 & 5,2 \\
\hline Fez uso de medicamentos para cólica?** & 520 & 100 & 299 & 100 & 221 & 100 \\
\hline Sim & 126 & 24,2 & 61 & 20,4 & 65 & 29,4 \\
\hline \multicolumn{7}{|c|}{$\begin{array}{l}0 \text { medicamento foi “dipirona sódica + cafeína } \\
\text { anidra + citrato de orfenadrina"?* }\end{array}$} \\
\hline Sim & 76 & 60,3 & 35 & 57,4 & 41 & 63,1 \\
\hline \multicolumn{7}{|l|}{ Quem indicou?* } \\
\hline a) Por conta própria & 24 & 31,6 & 07 & 20,0 & 17 & 41,5 \\
\hline b) Família/amigos & 21 & 27,6 & 10 & 28,6 & 11 & 26,8 \\
\hline c) M édico & 05 & 6,6 & 02 & 5,7 & 03 & 7,3 \\
\hline d) M eios de comunicação & 05 & 6,6 & 04 & 11,4 & 01 & 2,4 \\
\hline e) Não responderam & 21 & 27,6 & 12 & 34,3 & 09 & 22,0 \\
\hline
\end{tabular}

Fonte: Dados originais.

*Incluem os participantes que afirmaram "sim" na pergunta anterior; ${ }^{* *} 0$ total $(n=520)$ érepresentado por aqueles quefizeram uso de medicamentos nosúltimos sessenta dias. 
Tabela 4. Utilização e indicação de antimicrobianos por automedicação.

\begin{tabular}{|c|c|c|c|c|c|c|}
\hline \multirow{3}{*}{ Variáveis } & \multirow{2}{*}{\multicolumn{2}{|c|}{ Total }} & \multicolumn{4}{|c|}{ Dependência administrativa } \\
\hline & & & \multicolumn{2}{|c|}{ Públicas } & \multicolumn{2}{|c|}{ Privadas } \\
\hline & $\mathrm{n}$ & $\%$ & $\mathrm{n}$ & $\%$ & $\mathrm{n}$ & $\%$ \\
\hline Fez uso de antimicrobianos?* & 520 & 100 & 299 & 100 & 221 & 100 \\
\hline Sim & 51 & 9,8 & 32 & 10,7 & 19 & 8,6 \\
\hline \multicolumn{7}{|l|}{ Quem indicou o antimicrobiano?** } \\
\hline a) M édico & 22 & 43,1 & 11 & 34,4 & 11 & 57,9 \\
\hline b) Família/amigos & 22 & 43,1 & 16 & 50,0 & 06 & 31,6 \\
\hline c) Por conta própria & 07 & 13,8 & 05 & 15,6 & 02 & 10,5 \\
\hline
\end{tabular}

Fonte: Dados originais.

${ }^{*} 0$ total $(n=520)$ érepresentado por aqueles que fizeram uso de medicamentos nos últimos sessenta dias; ${ }^{* *}$ Inclui os participantes queafirmaram "sim" na pergunta anterior.

\section{Discussão}

No presente estudo, $72,0 \%$ dos estudantes utilizaram medicamentos nos últimos sessenta dias. Em estudo similar com crianças e adolescentes realizado nos municípios de Limeira e Piracicaba, no estado de São Paulo, a prevalência estimada de automedicação foi de $56,6 \%$. Ambas são superiores à prevalência de $49,7 \%$ encontrada por Arrais et al. ${ }^{17} \mathrm{em}$ um estudo que abordou 0 consumo de medicamentos em adultos no município deFortaleza. Esta diferença, no entanto, pode ser explicada pelo fato de que, nesta pesquisa, os participantes tinham idades entre trezee dezoito anos e o consumo de medicamentos foi avaliado considerando um período de sessenta dias, o que foi quatro vezes maior do que o relatado por outros autores que desenvolveram estudos em populações adultas.

Em outros estudos realizados por Bertoldi et al. ${ }^{18}$, em Pelotas, e por Vilarino et al. ${ }^{19}$, em Santa $M$ aria, encontrou-se maior consumo por parte do sexo feminino, o que também foi constatado nesta e nas demais pesquisas ${ }^{18-20}$.

A Lei no 5.991/73 define medicamento "como todo produto farmacêutico, tecnicamente obtido ou elaborado, com finalidade profilática, curativa, paliativa ou para fins de diagnósticos". A maioria dosalunos $(46,9 \%)$ definiu medicamento como "uma substância química com objetivo de cura", o que corrobora o dito por Lefèvre ${ }^{21} \mathrm{de}$ queéperfeitamente possível sever o medicamento como um signo cujo significado vai bem mais além da simples ideia, conceito de imagem mental de "saúde", implicando, também e, sobretudo, a própria realização e obtenção da saúde. A crença de que o medicamento simboliza a "saú- de" influencia as pessoas à prática da automedicação, porém, o risco encontra-se inerente a esse processo. N enhuma substância medicamentosa é inócua ao organismo, havendo para todas elas contraindicações e reações adversas, sendo 0 medicamento utilizado na forma correta ou de forma negligente, o que é outro fator agravante.

A pesar da grande diversidade das respostas, número significativo dos adolescentes $(17,0 \%)$ afirmou que as pessoas tomavam medicamentos sem receita médica porquejá possuíam experiência com o medicamento. Estudos evidenciam que aproximadamente $51,0 \%$ das decisões de se medicar são baseadas em prescrições anteriores ${ }^{22,23}$.

"A preguiça deir ao médico e/ou praticidade" e "dificuldadeno atendimento do SUS" foram respostasqueapresentaram diferenças significativas, em termos percentuais, entre os estudantes de escolas públicas e privadas, podendo tais diferenças ser justificadas pelas condições socioeconômicas de cada grupo. Percebe-se que a automedicação é praticada pelos dois grupos, mas por motivos opostos. 0 grupo de estudantes de escolas particulares, que, provavelmente, tem acesso à rede privada de saúde, não faz uso desta, por praticidade ou preguiça, praticando assim a automedicação. Alunos deescolas públicas, no entanto, que, possivelmente, são usuários do SUS, realizam tal prática, por terem dificuldades de atendimento no sistema público de saúde. Esta análise revela que as condições socioeconômicas podem interferir na frequência em quea automedicação ocorre, porém não restringem o exercício da automedicação a nenhuma classe social.

Constatou-sequemuitos adolescentes entendiam que se podem tomar medicamentos por conta própria, porém não justificaram tal asser- 
ção. Quanto aos riscos da automedicação, os estudantes relataram que sabiam ou sabiam parcialmente de tais riscos, mas grande parte não justificou a resposta. Esses dois fatos ressaltam a falta de informação e/ou orientação nesta temática, motivo relatado pelos estudantes como uma das razões para que as pessoas façam uso de medicamentos sem a prescrição médica.

As escolas aparecem como fonte de informação para $21,0 \%$, fato demonstrativo de que nenhuma das redes deensino está efetivamente tratando do tema transversal "saúde", definido nos Parâmetros Curriculares $\mathrm{N}$ acionais ${ }^{24}$ desde 1997, elaborado após a promulgação da Lei de Diretrizes eBases da Educação Nacional (LDB no 9.394/ 9.625) $)^{25}$. A falta de conhecimento por parte dos estudantes sobre o uso de medicamentos mostra quetanto a rede privada de ensino quanto a rede pública não dispõe de profissionais preparados para trabalhar as questões que envolvem a saúde, uma vez que a formação dos professores não contemplou aspectos relativos a estas temáticas, nem a escola conta em seu quadro com profissionais da saúde.

Diante disso, percebe seque sefaz necessário o desenvolvimento deações educativas em saúde na escola, no sentido deproporcionar ambientes educacionais mais saudáveis que promovam a saúde de todos os seus partícipes.

Os anal gésicos foram os medicamentos mais citados pelos adolescentes $(65,4 \%)$, fato que se mostrou semelhante a outros trabalhos desenvolvidos no país $s^{26-28}$. As classes de medicamentos encontradas já eram esperadas, já que os extremos etários são considerados os mai ores consumidores de medicamentos ${ }^{26}$, sendo crianças e idosos mais susceptíveis a enfermidades que necessitam de terapias medicamentosas específicas, as quais exigem maior controle da posologia e tempo de tratamento.

Constatou-se que houve mais casos de indicação dos medicamentos por parte dos familiares $(51,2 \%)$, seguido dos médicos (33,1\%) e por conta própria (20,8\%), sendo estas proporções obedecidas tanto para alunos das escolas particulares quanto para estudantes de escolas privadas. Diante destefato, percebe-sea necessidade de informação acerca do tratamento e de medicamentos por parte dos pacientes e familiares. Tal informação deve provir defontes fidedignas eatualizadas, o que, muitas vezes, não é possível apenas em bulários e propagandas ${ }^{29}$. A conscientização da comunidade é um pré requisito para que sejam alcançados níveis elevados de saúde ${ }^{27}$.

Considerando o fato de que os alunos de escolas particulares possuíam melhores condições socioeconômicas e ensino de melhor qualidade, entende-se o maior consumo de medicamentos por esses participantes, tanto por indicações médicas (38,9\%), como por automedicação (28,1\%) . 0 acúmulo deconhecimento ea disposição de maiores informações, que os auxiliam na escolha do medicamento, tornam o indivíduo mais confiante para se medicar ${ }^{28,29}$, porém a prática da automedicação também foi constatada em adolescentes da rede pública de ensino.

Apenas $29,1 \%$ dos alunos assinalaram já terem sido orientados sobre o uso correto de me dicamentos, sendo o médico (46,3\%) o mais referido pelas escolas públicas e os familiares $(47,4 \%)$, pelas escolas particulares. N otou-se a pequena participação do farmacêutico $(15,2 \%)$ nesse processo educativo, evidenciando que este profissional necessita conscientizar-se do seu papel na melhora da saúde pública, minimizando os riscos decorrentes da má utilização de medicamentos. Em estudo realizado por Arrais et al. ${ }^{30}$, apenas $25,8 \%$ de 833 pacientes receberam orientação sobre como tomar o medicamento no instante da dispensação.

A veiculação de campanhas publicitárias tendenciosas, cujos objetivos são meramente comerciais, estimula o consumo indiscriminado e crescentedemedicamentos ${ }^{31}$. Dos participantes, $34,1 \%$ fizeram uso de fármacos influenciados por meios de comunicação. Das alunas que tomaram medicamentos para cólica, 60,3\% fizeram uso do "dipirona sódica + cafeína anidra + citrato de orfenadrina", podendo haver relação entre 0 alto índice de uso e a propaganda televisionada deste produto, que ocorria durante a coleta de dados do presente estudo. A propaganda se torna perigosa, levando em conta a ideia de que induz à automedicação por pessoas que não têm atitude crítica na recepção da publicidade demedicamentos para reconhecer o limite do seu uso dentro da atenção à saúde 5 . Quando questionados sobre quem indicou o medicamento "dipirona sódica + cafeína anidra + citrato de orfenadrina", apenas $6,6 \%$ responderam haver sido os meios de comunicação, porém $59,2 \%$ revelaram que foram a família, os amigos ou automedicação, o que permite questionar-se sobre a influência da mídia.

0 fato de medicamentos serem vendidos em locais inadequados foi detectado por este estudo. Os estudantes de escola pública relataram, em maior proporção $(25,8 \%)$, o fato de que conseguiam medicamentos sem receita médica em supermercados emercearias, enquanto apenas 2,6\% dos alunos de escolas particulares relataram tal prática. A dispensação de medicamentos é privativa dos estabelecimentos autorizados e definidos 
pela Lei $n^{0} 5.991^{32}$, especificando farmácias, drogarias, postos de medicamento, unidades volantesedispensário demedicamentos. Segundo M elo et al. ${ }^{33}$, a venda de medicamentos anódinos em supermercados e demais locais leigos pode engrossar as estatísticas de incidência de intoxicações medicamentosase somente com umafiscalização sanitária atuante, associada à educação da população, os perigos da automedicação poderiam tomar um rumo menos desastroso.

\section{Considerações finais}

Ao final do estudo, é possível concluir que a automedicação entre os adolescentes de escolas públicas e privadas deFortaleza (CE) ocorresem controle sanitário, familiar e institucional.

0 conhecimento dos adolescentes sobre medicamento e suas implicações na saúde mostrou-se incipiente e desprovido de qualquer noção básica sobre o uso racional demedicamento. Sua concepção sobremedicamento tem sido influenciada pelo modelo biomédico: "combater sintomas" e "prevenir doenças", reflexo da visão descontextualizada do tema saúde nos espaços educacionais.

Chama aten ção o fato de estudantes da rede pública receberem mais orientações sobre o uso correto de medicamentos por parte dos profissionais do sistema público de saúde, demonstrando a eficiência do sistema como promotor de saúde da população matriculada em escolas públicas de Fortaleza e a não utilização do sistema público de saúde pelos estudantes da rede particular de ensino.

$N$ as duas realidades educacionais, énotória a banalização da prática da automedicação na adolescência, particularmente entre as meninas, vislumbrando-se possíveis usuárias permanentes de medicamentos.

\section{Colaboradores}

IM Silva, AM F Catrib, VC de M atos e APS Gondim participaram igualmente de todas as etapas da elaboração do artigo. 
1. Carvalho RAS. U so racional de medicamentos: subsídios para uma proposta de ação educativa em saúde [dissertação]. Fortaleza (CE): Universidade de Fortaleza; 2003.

2. Sociedade Brasileira de Vigilância de M edicamentos. 0 que é uso racional de medicamentos. São Paulo: Sobravime; 2001.

3. Berquó LS, Barros AJD, Lima RC, Bertoldi AD. Utilização de medicamentos para tratamento de infecções respiratórias na comunidade. Rev. Saude Publica 2004; 38(3):358-364.

4. Lefévre F. O medicamento como mercadoria simbólica. São Paulo: Cortez; 1991.

5. Lage EA, Freitas MIF, Acurcio FA. Informação sobre medicamentos na imprensa: uma contribuição para o uso racional? Cien Saude Colet 2005; 10(Supl.):133-139.

6. Lima GB, De Araujo EJF, Sousa KMH, Benvido RF, Silva WC, Correa Jr. RAC, Nunes LCC. Avaliação da utilização de medicamentos armazenados em domicílios por uma população atendida pelo PSF. Rev. Bras. Farm. 2008; 89(2):146-149.

7. Mota DM, Da Silva M GC, Sudo EC, Ortún V. Uso racional de medicamentos: uma abordagem econômica para tomada de decisões. Cien Saude Colet 2008; 13(Supl.):589-601.

8. Organización Mundial de la Salud. Promoción del uso racional de medicamentos: componentes centrales. [site da Internet] 2002 [acessado 2007 out 23]. Disponível em: http://whqlibdoc.who.int/hq/2002/ WHO_EDM_2002.3_spa.pdf

9. Béria JU, Victora CG, Barros FC, Teixeira AB, Lombardi $C$. Epidemiologia do consumo de medicamentos em crianças de centro urbano da região sul do Brasil. Rev. Saude Publica 1993; 27:95-104.

10. Urbano B, Magro R, Masip M, Vacas R. Automedicación en pediatría general. Atención Primaria 1994; 13:313-316.

11. Bricks LF, Leone C. Utilização de medicamentos por crianças atendidas em creches. Rev. Saude Publica 1996; 30:527-535.

12. Weiderpass E, Béria JU, Barros FC, Victoria CG, Tomasi E, Halpern R. Epidemiologia do consumo de medicamentos no primeiro trimestre de vida em centro urbano do sul do Brasil. Rev. Saude Publica 1998; 32:335-344.

13. Gomes MFS. Estudo da automedicação infantil em uma região administrativa no município do Rio de Janeiro [dissertação]. Rio de Janeiro (RJ): Universidade Federal do Rio de Janeiro; 2000.

14. Bricks LF. Uso judicioso de medicamentos em crianças. J Pediatr (Rio J) 2003; 79(Suppl. 1):S107-S114.

15. Pereira FS, Bucaretchi F, Stephan C, Cordeiro R. Self-medication in children and adolescents. J $\mathrm{Pe}$ diatr (Rio J) 2007; 83(5):453-458.

16. Brasil. M inistério da Saúde. Conselho Nacional de Saúde. Resolução n 196 de 10 de outubro de 1996. A prova as diretrizes e normas regulamentadoras de pesquisa envolvendo seres humanos. Diário Oficial da União 1996; 16 out.

17. Arrais PSD, Brito LL, Barreto ML, Coelho HLL. Prevalência e fatores determinantes do consumo de medicamentos no M unicípio de Fortaleza, Ceará, Brasil. Cad Saude Publica 2005; 21(6):1737-1746.
18. Bertoldi AD, Barros AJD, Hallal PC, Lima RC. Utilização de medicamentos em adultos: prevalência e determinantes individuais. Rev. Saude Publica 2004; $38(2): 228-238$.

19. Vilarino JF, Soares IC, Silveira CM, Rodel APP, Bortoli R, Lemos RR. Perfil da automedicação em município do sul do país. Rev. Saude Publica 1998; 32(1):43-49.

20. Arrais PSD, Coelho HLL, Batista MCDS, Carvalho $M L$, Righi RE, Arnau JM. Perfil da automedicação no Brasil. Rev. Saude Publica 1997; 31(1):71-77.

21. Lefèvre $F$. A oferta e a procura de saúde através do medicamento: proposta de um campo de pesquisa. Rev. Saude Publica 1987; 21(1):64-67.

22. Loyola AIF, Uchoa E, Guerra HL, Firmo JOA, Lima MFC. Prevalência e fatores associados à automedicação: resultados do projeto Bambuí. Rev. Saude Publica 2002; 36(1):55-62.

23. Volpato DE, Souza BV, Dalla R, Melo LH, Daudt CAS, Deboni I. Use of antibiotics without medical prescription. Braz J Infect Dis 2005: 9(4):288-291.

24. Brasil. Ministério da Educação e do Desporto. Secretaria do Ensino Fundamental. Parâmetros curriculares Nacionais. Brasília: MEC; 1997.

25. Brasil. Ministério da Educação e do Desporto. Lei no 9.394 de 20 de dezembro de 1996. Dispõe sobre as diretrizes e bases da educação nacional. Diário Oficial da União 1996; 20 dez.

26. Nitschke CAS, Guimarães FS, Cunha J, Dutra ACA, Silva Júnior M M. Estudo Sobre o uso de medicamentos em quatro bairros de Porto Alegre. Rev AM RIGS 1981; 25(1):184-189.

27. Melo DO, Ribeiro E, Storpitis S. A importância e a história dos estudos de utilização de medicamentos. Rev. Bras Cienc. Farm. 2006; 42(4):475-485.

28. Vieira FS. Possibilidades de contribuição do farmacêutico para a promoção da saúde. Cien Saude Colet 2007; 12(1):213-220.

29. Saeed AA. Self-medication among primary care patients in Farazdak Clinic in Riyadh. Soc Sci Med 1988; 27(1):287-289.

30. Arrais PSD, Barreto ML, Coelho HLL. Aspectos dos processos de prescrição e dispensação de medicamentos na percepção do paciente: estudo de base populacional em Fortaleza, Ceará, Brasil. Cad Saude Publica 2007; 23(4):927-937.

31. Luchessi AD, Marçal BF, Araújo GF, Uliana LZ, Rocha MRG, Pinto TJA. M onitoração de propaganda e publicidade de medicamentos: âmbito de São Paulo. Rev. Bras Cienc. Farm. 2005; 41(3):345-349.

32. Brasil. Ministério da Saúde. Lei $n^{\circ} 5.991$ de 17 de dezembro de 1973. Dispõe sobre o controle sanitário do comércio de drogas, medicamentos, insumos farmacêuticos e correlatos, e dá outras providências. Diário Oficial da União 1973; 17 dez.

33. M elo EB, Teixeira JJVM, Graciele CM. Histórico das tentativas de liberação da venda de medicamentos em estabelecimentos leigos no Brasil a partir da implantação do Plano Real. Cien Saude Colet 2007; 12(5):1333-1339.

Artigo apresentado em 26/11/2008

Aprovado em 28/08/2009

Versão final apresentada em 12/11/2009 